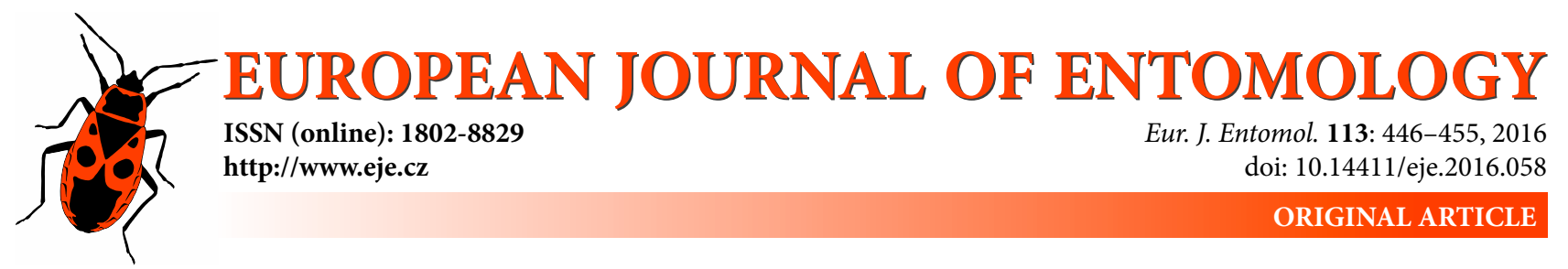

\title{
Genetic identification of a non-native species introgression into wild population of the field cricket Gryllus campestris (Orthoptera: Gryllidae) in Central Europe
}

\author{
Hanna PANAGiotopoulou ${ }^{1,2, *}$, Mateusz BACA ${ }^{3, *}$, Katarzyna BACA $^{4}$, PaWek SiENKIEWICZ ${ }^{5}$, Piotr ŚliPińSKI ${ }^{1}$ \\ and MıcHAŁ ŻMIHORSKI ${ }^{6,7, * *}$ \\ ${ }^{1}$ Museum and Institute of Zoology, Polish Academy of Sciences, Wilcza 64, 00-679 Warsaw, Poland; \\ e-mails: hpana@miiz.waw.pl, piotrs@miiz.waw.pl \\ ${ }^{2}$ Institute of Biochemistry and Biophysics, Polish Academy of Sciences, Pawińskiego 5a, 02-106 Warsaw, Poland \\ ${ }^{3}$ Center for Precolumbian Studies, University of Warsaw, Krakowskie Przedmieście 26/28, 00-927 Warsaw, Poland; \\ e-mail: bacamat@gmail.com \\ ${ }^{4}$ Institute of Genetics and Biotechnology, Department of Biology, University of Warsaw, Pawińskiego 5a, 02-106 Warsaw, \\ Poland; e-mail: katarzyna.ewa.pajak@gmail.com \\ ${ }^{5}$ Department of Entomology and Environmental Protection, Poznań University of Life Sciences, Dąbrowskiego 159, \\ 60-594 Poznań, Poland; e-mail: ophonus@gmail.com \\ ${ }^{6}$ Institute of Nature Conservation, Polish Academy of Sciences, Mickiewicza 33, 31-120 Kraków, Poland \\ ${ }^{7}$ Department of Ecology, Swedish University of Agricultural Sciences, Box 7044, SE 75007 Uppsala, Sweden
}

Key words. Orthoptera, Gryllidae, Gryllus campestris, Gryllus bimaculatus, conservation, mtDNA, microsatellite loci, $\mathrm{X}$-chromosome-linked markers

\begin{abstract}
Two species of the genus Gryllus occur in Europe: G. campestris and G. bimaculatus. The first is widely distributed in the north-western Palaearctic, while the second, G. bimaculatus, occurs predominantly in the Mediterranean area. There is a visible pattern in the distribution of G. campestris, the insect being rare and threatened in the western part of its range, whereas it is still abundant in the east. Despite the fact that this species is commonly used in laboratory experiments, its natural populations are poorly characterised. In the present study, we analysed cricket populations from the lower Oder and Vistula River valleys in Poland. Based on the phylogeny of the mtDNA cytochrome $b$ fragment, we found that $17 \%$ of the individuals studied had a G. bimaculatus-like mtDNA haplotype. Analyses of 11 autosomal microsatellite loci failed to reveal any clear genetic differentiation between individuals assigned to these two clades. This suggests, along with the spatial distribution of G. bimaculatus-like haplotypes, successful interbreeding of G. bimaculatus with native populations of $G$. campestris. However, both the nuclear data and additional analyses of two X-chromosome-linked microsatellite loci revealed incomplete introgression. Human-mediated introgression seems to be the most plausible explanation of the observed genotypic pattern such that caution needs to be taken in conservation efforts carried out in the western part of the species' range.
\end{abstract}

\section{INTRODUCTION}

Populations of the field cricket, Gryllus campestris L. (Orthoptera: Gryllidae) vary in terms of conservation status across the species' European range. In the UK, Germany, Netherlands, Denmark and Switzerland, the species is rare and included in the Red Lists (Hochkirch et al., 2007). In contrast, populations from central-eastern Europe are abundant, and appear to show stable dynamics over time (Bazyluk \& Liana, 2000). The most distinct contrast in the state of the field cricket population seems to be visible in Germany and Poland. Grein (2000, 2005 after Hochkirch et al., 2007) states that only ten populations are left in Lower Saxony and one west of the Weser River. At the same latitude, but $400 \mathrm{~km}$ to the east in Poland, the species is still very common and widely distributed throughout the whole country (Bazyluk \& Liana, 1990, 2000).

This contrast between populations in the west and east of Europe may be the result of differences in land-use man-

\footnotetext{
* These authors equally contributed to the work.

** Corresponding author; e-mail: michal.zmihorski@gmail.com
} 
agement, landscape history and agriculture intensification (see Table 2 in Tryjanowski et al., 2011). Studies conducted on various Orthoptera species in the Czech Republic suggest habitat loss, due to changes in land management and forestations, as a predominant factor that has resulted in considerable fragmentation and isolation of many invertebrate populations (Holusa, 2012; Holusa et al., 2012). The decline of G. campestris in Western Europe is likely to be due to isolation of small populations accelerating inbreeding and the associated increased extinction risk (Witzenberger \& Hochkirch, 2008). Nevertheless, knowledge concerning the status and temporal trends in population dynamics of the field cricket in different locations across its European distribution range is still poor. While the species is commonly used in laboratory studies, its natural populations are rarely investigated (but see e.g. Ritz \& Kohler, 2007; Witzenberger \& Hochkirch, 2008; Rodríguez-Muñoz et al., 2010; Bretman et al., 2011). Even basic ecological parameters are unknown, and there is no reliable data on the genetic diversity of the species, especially in the eastern part of its range.

The distribution of G. campestris includes all of Europe and overlaps with the distribution of its sister species, $G$. bimaculatus De Geer towards the south (Fig. 1). The latter species' range in Europe is mainly restricted to the Mediterranean coastal regions. Both species share common life histories, utilizing similar habitats and have recently been studied for patterns of reproductive barrier development that may have caused their speciation and prevent hybridization (Tyler et al., 2013; Veen et al., 2011, 2013). Contact zones between these two field cricket species exist in south-eastern Spain and probably also in Italy and further east, although geographical barriers like the Cantabrian Mountains probably prevent secondary contact (Bazyluk \& Liana, 2000; Veen et al., 2013 and references therein). In contact zones of the two species, the possibility of introgression exists, and it has indeed been experimentally demonstrated that they can hybridize (Veen et al., 2011, 2013; Tyler et al., 2013). The intensity of inter-specific crossing in the natural environment remains unknown, though earlier studies suggest that gene flow between these two species ought to be highly restricted via multiple reproductive barriers (Veen et al., 2013).

The natural ability of field crickets to hybridize has received considerable attention as a very good model to investigate evolutionary forces that promote and maintain speciation. The topic has also been recently intensively studied using molecular (DNA) markers (Harrison, 1983; 1986; Harrison et al., 1987; Maroja et al., 2009, 2014; Veen et al., 2011, 2013; Larson et al., 2012; Andrés et al., 2013; Tyler et al., 2013). These studies aimed to discover reproductive barriers acting at pre- and post-mating stages. It was proven that prezygotic barriers between G. campestris and G. bimaculatus are strong and include pre-mating isolation via sexual signals and selection (based on calling song preferences and probably species-specific cuticular hydrocarbons) as well as post-mating prezygotic conspecific sperm precedence and cryptic female choice (Veen

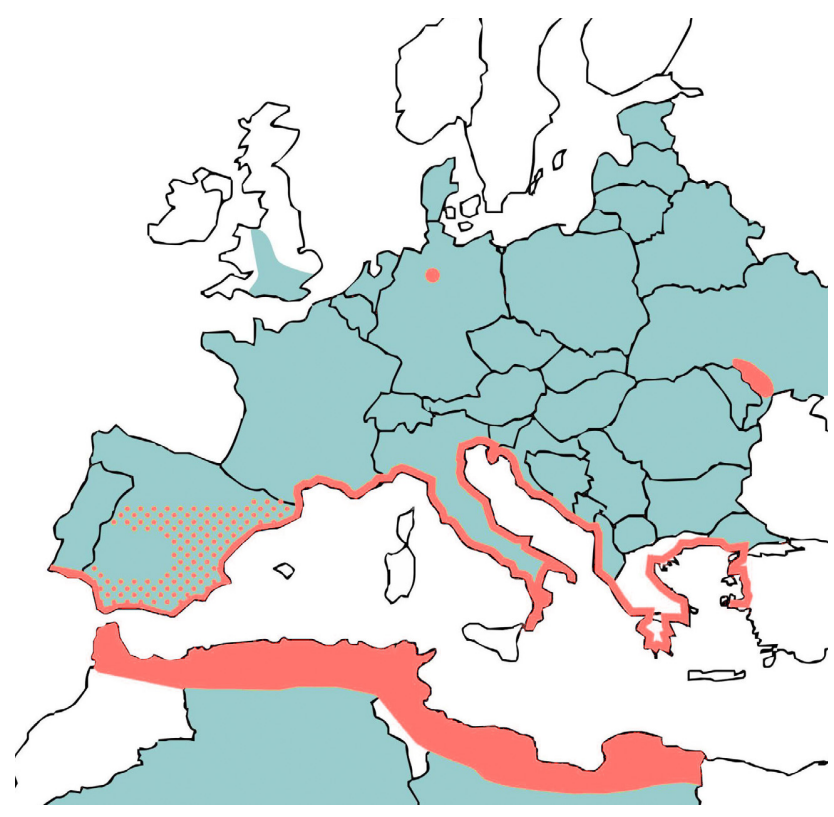

Fig. 1. Schematic distribution of the two cricket species in CentralWestern Europe: Gryllus campestris (blue) and G. bimaculatus (pink).

et al., 2011, 2013; Tyler et al., 2013). Post-zygotic barriers between these two species seem to be much weaker, though hybrid offspring are negatively sexually selected and have lower fitness. This includes reduced hatching success, which is probably a result of higher mortality in the very early life stages (Veen et al., 2013). Hybrids do however live longer (Veen et al., 2013), which makes their life-time reproductive success higher (Rodríguez-Muñoz et al., 2010). This incomplete reproductive isolation suggests the possibility of successful introgression in the wild in areas where both species coexist naturally or come into secondary contact due to human introductions.

In the present study, we have attempted to characterize the genetic polymorphism and structure of wild G. campestris populations in the eastern part of the species' distribution range. More specifically, we studied both mitochondrial as well as nuclear genetic markers of 488 crickets captured in the wild. During the research we discovered introgression of G. bimaculatus-like haplotypes into the $G$. campestris gene pool. Possible explanations for the occurrence of G. bimaculatus' far outside its natural distribution and the possible consequences for conservation of natural populations of $G$. campestris are discussed.

\section{MATERIALS AND METHODS}

\section{Sampling}

In total 414 individuals were collected in the lower Oder River valley of north-western Poland $\left(52.89^{\circ} \mathrm{N}, 14.32^{\circ} \mathrm{E}\right)$ in May-June, 2010 and 2011 in 21 different localities within this region. These particular sampling sites, covering different types of habitats utilized by the species - xerothermic grasslands, fields, meadows and clear-cut areas in the forest complex - were selected in order to characterize genetic structure of the populations living under different environmental conditions. Additionally, we collected 74 individuals from the lower Vistula River valley $\left(53.05^{\circ} \mathrm{N}\right.$, $18.40^{\circ} \mathrm{E}$ ) at three localities on the basis of visual and aural detection in appropriate habitats. The trapped animals were kept in 
captivity until their natural death, which took place in AugustSeptember, and afterwards they were preserved in $75 \%$ ethanol.

\section{DNA extraction, amplification and sequencing}

DNA was isolated from one rear leg of each specimen using the Wizard SV 96 Genomic DNA Purification System (Promega, Madison, WI, USA) according to the manufacturer's recommendations. In order to determine species affiliation of Gryllus specimens, a 846 bp region of the cytochrome $b$ sequence was amplified with primers 846 For 5'-AGA GTA AGT CAC ATT TGC CGA G-3' and 846Rev 5'-GGT TCT TCA ACT GGT CGG GCA CC-3' designed with Primer 3 v.0.4.0 software (Untergasser et al., 2012). PCR reactions were performed in $20 \mu$ of reaction mix containing $0.2 \mu \mathrm{M}$ of each primer, $1 \times$ Biomix (Bioline, London, UK), and 1 or $2 \mu 1$ of extracted DNA. Initial denaturation of 5 min at $95^{\circ} \mathrm{C}$ was followed by 31 cycles of $30 \mathrm{~s}$ at $95^{\circ} \mathrm{C}, 30 \mathrm{~s}$ at $58^{\circ} \mathrm{C}, 1 \mathrm{~min}$ at $72^{\circ} \mathrm{C}$ followed by a final extension of $7 \mathrm{~min}$ at $72^{\circ} \mathrm{C}$. PCR products were purified using PEG precipitation and sequenced. DNA chromatograms were analyzed using FinchTV v.1.4.0 (Geospiza, Seattle, WA, USA), and aligned with BioEdit software v.7.0.5.3 (Hall, 1999).

In addition to the mitochondrial marker, 14 microsatellite loci, 12 autosomal and two sex-linked i.e. Gbim59 and Gbim 71 (Dawson et al., 2003; Bretman et al., 2008) were amplified, divided into 4 multiplex reactions (Panagiotopoulou et al., 2015). Capillary electrophoresis of PCR products, divided into two panels, was conducted in Oligo.pl service (IBB, Warsaw, Poland) on an ABI PRISM 3730 Genetic Analyzer (Applied Biosystems, Carlsbad, CA, USA). The Peak Scanner program v.1.0 (Applied Biosystems) was used to bin, score and output the microsatellite alleles.

\section{Phylogenetic analyses}

A phylogenetic tree was reconstructed to confirm species affiliation of the studied individual crickets. Cytochrome $b$ sequences of two Gryllus species that are common in Europe, G. campestris and its sister species G. bimaculatus, were obtained from the literature (Huang et al., 2000; Fereira \& Ferguson, 2010). Phylogenetic analyses were based on 575 bp long sequences. Phylogenetic trees were constructed using three approaches: (1) Maximum likelihood (ML) and (2) Neighbour Joining (NJ) trees were constructed in MEGA v.5 software (Tamura et al., 2011) with 1,000 bootstrap replicates used for each analysis. TamuraNei with a proportion of invariable sites (TN93 + I) was chosen as a best fitting substitution model with MEGA v.5 software and used for NJ and ML analyses. In addition, a Bayesian tree (3) was reconstructed in MrBayes v.3.2.3 (Ronquist \& Huelsenbeck, 2003). We used a nst=mixed option to allow MrBayes to sample over all possible reversible substitution models. We applied gamma distributed rate variation across sites and allowed for proportion of invariable sites. Different substitution rates for each codon position were allowed. Two independent runs with four Markov chains each were run for 10,000,000 generation with parameters sampled every 1,000 generations. Runs were checked for stationarity and convergence using the program Tracer v. 1.6 (ESS above 200 and the standard deviation of split frequencies lower than 0.01).

\section{Spatial distribution of individuals carrying the two cytochrome $b$ haplotypes}

To check, whether the G. bimaculatus-like cytochrome $b$ haplotype was randomly distributed in the study area, multiple logistic regression was used. The share of G. bimaculatus-like haplotype among all crickets sampled in a given location was modeled as a function of latitude and longitude and presented on the map in form of pie plots. The analysis was performed in R (R Development Core Team, 2015).

\section{Genetic polymorphism and structure estimations}

In order to verify the amount of gene flow - introgression or reproductive separation between the two detected mitochondrial lineages of crickets, we applied a microsatellite screening approach. For this, only individuals whose cytochrome $b$ haplotype was identified were used. Micro-Checker v.2.2.3 software (van Oosterhout et al., 2004), was applied, using 1,000 iterations and $95 \%$ CI, to search for genotyping errors caused by false alleles, stuttering, null alleles (assuming the Brookfield 1 equation) and allelic dropouts in cricket groups from each sampling location treated separately. Locus Gbim58 was discarded from further analyzes, because it possessed a high and significant frequency of null alleles $(>0.19)$ in nearly all of the analyzed populations. As loci Gbim 59 and Gbim 71 were shown to be linked to the chromosome X (Bretman et al., 2011), they were analyzed separately.

The observed allelic range, number of alleles per locus $\left(N_{A}\right)$ and effective number of alleles $\left(N_{e}\right)$ were calculated using GeneAlEx v.6.4 software (Paekal \& Smouse, 2006). Observed heterozygosity $\left(H_{O}\right)$ and expected heterozygosity $\left(H_{E}\right)$ were obtained with Arlequin v.3.5.1.2 (Excoffier \& Lischer, 2010). Allelic richness $(R)$ and inbreeding coefficients $\left(F_{I S}\right)$ were estimated in FSTAT v.2.9.3.2 (Goudet, 2002). Deviation from Hardy-Weinberg equilibrium (HWE) was tested using Genepop v.4.0.10 (Raymond \& Rousset, 1995).

Genetic distances between pairs of individuals were calculated using the $D_{A}$ measure (Nei, 1983) with MSA software v.4.05 (Dieringer \& Schlötterer, 2003). The obtained values matrix of $D_{A}$ values was visualized using principal coordinate analysis (PCoA) implemented in GeneAlEx. The locations of individuals belonging to the two haplotypes were visualized with the kernel density estimator plotted along the first two axes of the PCoA. It was checked, moreover, whether or not the two haplotypes ( $G$. campestris and G. bimaculatus-like) were separable along the PCoA axes. More specifically, we compared the mean values of all PCoA axes scores $(\mathrm{n}=123$ axes) for $G$. bimaculatus-like and G. campestris individuals with $t$-tests for independent samples.

Genetic structure was analyzed with the Bayesian approach implemented in STRUCTURE v.2.3.4 (Pritchard et al., 2000). Simulations assumed admixture, no a priori population information, and the correlated allele frequency model. Ten clusters $(K)$ from 1 to 10 were inferred. In each case, the program was run with ten repetitions of 500,000 MCMC iterations following a burn-in period of 50,000 iterations. The most probable number of clusters $(K)$ was determined in the Structure Harvester v.0.6.8 program (Earl \& von Holdt, 2012) by calculating $\Delta K$ as described by Evanno et al. (2005). The average coefficients of membership $(Q)$ of individuals to each of the pre-defined clusters across the ten repetitions were computed using CLUMPP v.1.1.2 (Jakobbson \& Rosenberg, 2007) and displayed with the DISTRUCT v.1.1 (Rosenberg, 2004) software. Next, the relationship between the genetic structure of the whole population and assignment of the crickets to the two mitochondrial groups ( $G$. bimaculatus-like vs. G. campestris) was investigated. Here, we used the chi-square test $(2 \times 2$ contingency table) in order to check whether assignment of the crickets into one of the two mitochondrial groups depended on its classification to a particular cluster according to the results obtained using STRUCTURE.

\section{RESULTS}

\section{Phylogenetic analyses}

Three distinct haplotypes were discovered in the Gryllus dataset. Phylogenetic analyses showed that two of them (H1 and H2) grouped with G. campestris haplotypes. The third 


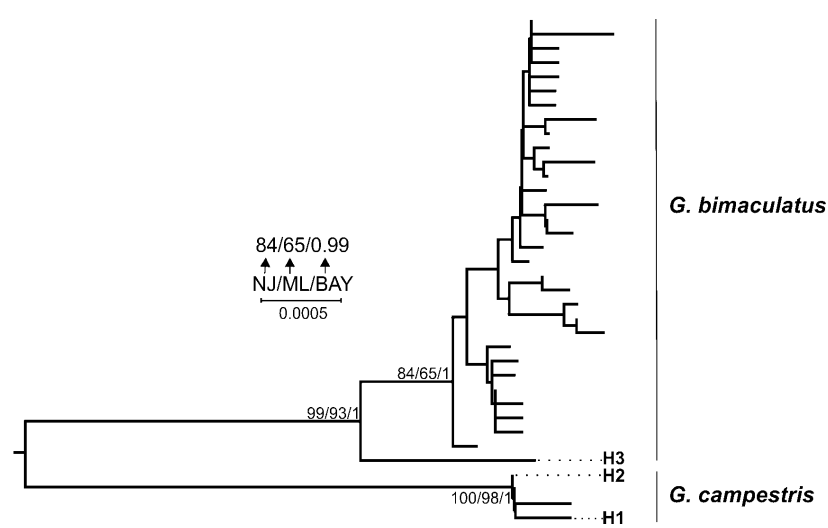

Fig. 2. Phylogenetic tree based on a 575 bp of cytochrome $b$ sequences. The position of the haplotypes obtained in this study $(\mathrm{H} 1-\mathrm{H} 3)$ is as shown. Bootstrap support and posterior probabilities of main nodes are indicated. The outgroup Acheta domestica (L.) (Orthoptera: Gryllidae) was removed for display purposes.

haplotype (H3) was located on the G. bimaculatus branch, although it was highly divergent from all G. bimaculatus specimens (Fig. 2). The position of this haplotype was consistent across all tree reconstruction methods used with high bootstrap and posterior probability values. Specimens carrying haplotype $\mathrm{H} 3$ could not be easily assigned to $G$. bimaculatus; however, since available cytochrome $b$ sequence data of $G$. bimaculatus is limited to South African and three western Mediterranean populations, we cannot therefore exclude the possibility that this haplotype came from a divergent near Eastern or Asiatic subpopulation of this species. The recorded haplotypes were deposited in GenBank under accession no. KF182315-17, along with the computed trees that were uploaded to TreeBase database (accession no. S19499).

Haplotype H3 was present in 72 of 391 individuals from the lower Oder River and absent in the lower Vistula River valley sampling localities (i.e. of 23 individuals tested) and the difference in the observed frequencies (i.e. 72 vs. 319 and 0 vs. 23$)$ was significant $(2 \times 2$ contingency table chisquare test with Yates correction, $\left.\chi^{2}=3.92 ; P=0.048\right)$. At a lower spatial scale, a clear and statistically significant east-west frequency gradient of G. bimaculatus-like haplotypes was observed in the lower Oder River valley region (multiple logistic regression, estimate $=3.19, \mathrm{t}=3.61, P=$ $0.0019)$. This haplotype had a frequency of $\leq 75 \%$ of individuals in populations from the east and their frequency decreased towards the western part of the sampling area (Fig. 3). The effect of latitude was not significant (estimate $=1.77, \mathrm{t}=0.82, P=0.4216)$.

\section{Populations' genetic polymorphism}

Analyses of the 11 autosomal microsatellite markers showed a very similar level of polymorphism and nearly identical allelic size range across the 414 individuals tested, when the two groups of crickets representing G. campestris $(\mathrm{n}=342)$ and $G$. bimaculatus-like $(\mathrm{n}=72)$ haplotypes were compared (Table 1). The mean number of alleles $\left(N_{A}\right)$ and allelic richness $(R)$ were however slightly lower for the group of individuals with the $G$. bimaculatus-like haplotype, which indicates intermixing of a small group into a bigger gene pool. In the case of the $N_{A}$ values, this difference results mainly from the larger sample size of the G. campestris group. Despite this, all other differences in diversity indices between the two crickets groups were not

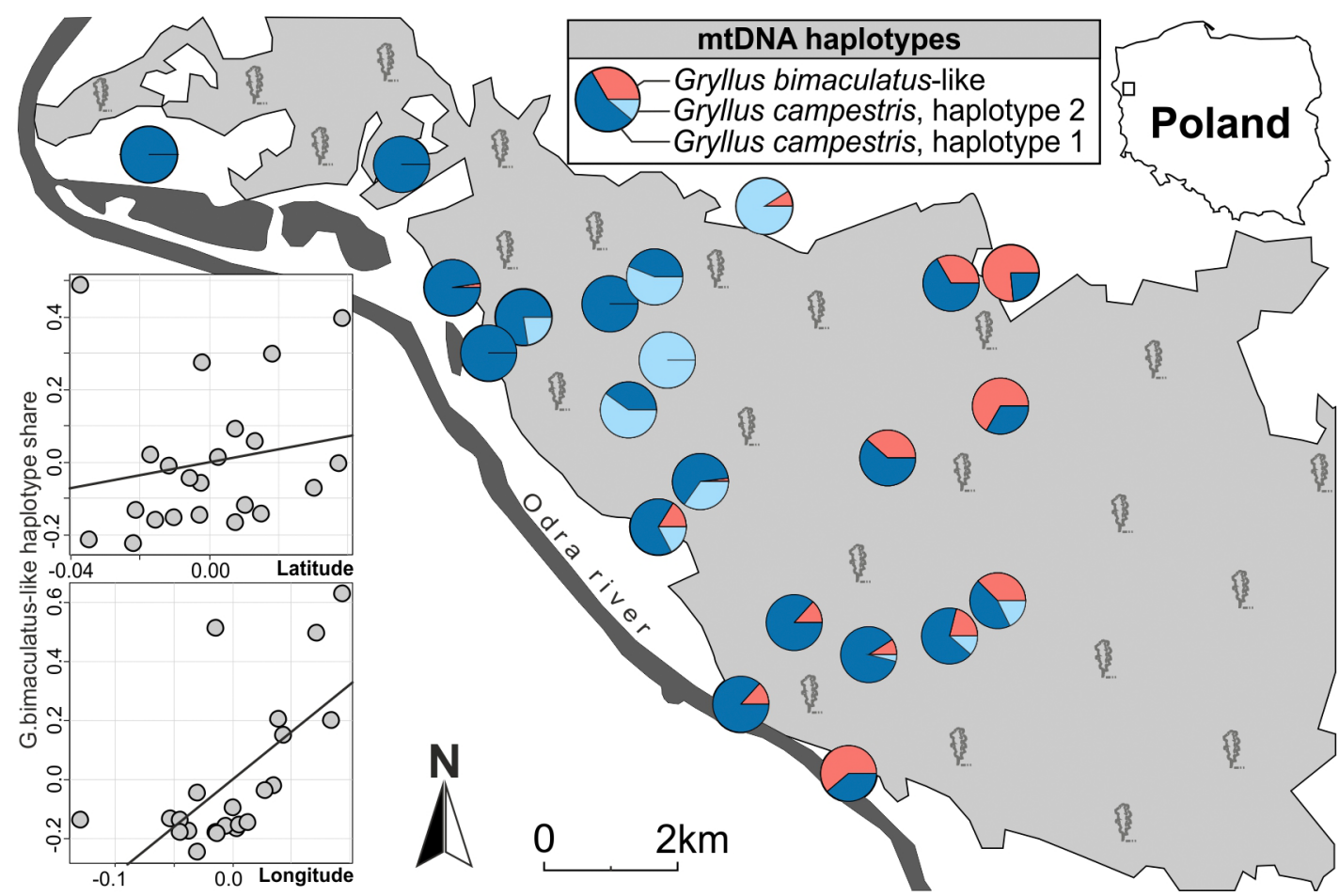

Fig. 3. Share of G. campestris and G. bimaculatus-like specimens within the sampled populations from the Oder River valley region. Visualization of the partial effects of longitude and latitude is given in the inner subplot - the longitude is a significant predictor for the sharing of G. bimaculatus-like haplotypes. The pale grey polygon indicates forested areas, dark grey - water bodies. 
TABLE 1. Summary of the allelic variation and gene diversity indices at 11 autosomal microsatellite markers in the two field crickets groups representing $G$. campestris $(\mathrm{n}=342)$ and $G$. bimaculatus-like $(\mathrm{n}=72)$ haplotype lineages. $N_{\mathrm{A}}$ - number of alleles; $N_{\mathrm{e}}$ - effective number of alleles; $R$ - allelic richness; $H_{0}-$ observed heterozygosity; $H_{E}$ - expected heterozygosity; $F_{I S}$ - inbreeding coefficient; HWE - deviation from Hardy-Weinberg equilibrium estimated from $F_{I S}$ values: * - significant $F_{I S}$ values at a probability level equivalent to $P<0.05$ and NS - not significant $F_{I S}$ values. Significance obtained from paired samples $t$-test are given in the last column.

\begin{tabular}{|c|c|c|c|c|c|c|c|c|c|c|c|c|c|c|}
\hline Measure & Cricket group & Gbim15 & Gbim35 & Gbim21 & Gbim40 & Gbim72 & Gbim29 & Gbim57 & Gbim33 & Gbim49 & Gbim04 & Gbim66 & Mean $\mathrm{F}$ & P-value \\
\hline \multirow[t]{2}{*}{$N_{A}$} & G. camprestris & 9 & 8 & 27 & 14 & 7 & 12 & 7 & 18 & 4 & 6 & 10 & 11.09 & \multirow{2}{*}{0.014} \\
\hline & G. bimaculatus-like & 9 & 3 & 16 & 12 & 5 & 11 & 5 & 15 & 3 & 6 & 6 & 8.27 & \\
\hline \multirow[t]{2}{*}{$N_{e}$} & G. camprestris & 4.5 & 1.7 & 10.6 & 2.9 & 3.2 & 7.6 & 1.5 & 5.5 & 3.0 & 4.1 & 3.1 & 4.33 & \multirow{2}{*}{0.741} \\
\hline & G. bimaculatus-like & 4.0 & 1.4 & 7.4 & 4.5 & 2.3 & 6.9 & 1.4 & 7.9 & 2.9 & 3.9 & 3.5 & 4.21 & \\
\hline \multirow[t]{2}{*}{$R$} & G. camprestris & 8.6 & 4.8 & 21.8 & 11.4 & 5.4 & 10.8 & 5.5 & 14.6 & 3.9 & 6.0 & 6.7 & 9.05 & \multirow{2}{*}{0.133} \\
\hline & G. bimaculatus-like & 8.9 & 3.0 & 16.0 & 11.7 & 4.9 & 10.9 & 5.0 & 14.8 & 3.0 & 5.8 & 6.0 & 8.18 & \\
\hline \multirow[t]{2}{*}{$H_{0}$} & G. camprestris & 0.68 & 0.06 & 0.57 & 0.58 & 0.64 & 0.73 & 0.31 & 0.71 & 0.34 & 0.64 & 0.59 & 0.53 & \multirow{2}{*}{0.240} \\
\hline & G. bimaculatus-like & 0.74 & 0.12 & 0.54 & 0.70 & 0.60 & 0.79 & 0.29 & 0.79 & 0.45 & 0.54 & 0.58 & 0.56 & \\
\hline \multirow[t]{2}{*}{$H_{E}$} & G. camprestris & 0.78 & 0.41 & 0.91 & 0.65 & 0.69 & 0.87 & 0.34 & 0.82 & 0.67 & 0.76 & 0.68 & 0.69 & \multirow{2}{*}{0.623} \\
\hline & G. bimaculatus-like & 0.76 & 0.31 & 0.87 & 0.78 & 0.56 & 0.86 & 0.31 & 0.88 & 0.66 & 0.75 & 0.72 & 0.68 & \\
\hline \multirow[t]{2}{*}{$F_{I S}$} & G. camprestris & 0.12 & 0.85 & 0.37 & 0.12 & 0.07 & 0.16 & 0.07 & 0.13 & 0.49 & 0.15 & 0.13 & 0.23 & \multirow{2}{*}{0.146} \\
\hline & G. bimaculatus-like & 0.03 & 0.61 & 0.38 & 0.10 & -0.06 & 0.09 & 0.06 & 0.11 & 0.32 & 0.28 & 0.19 & 0.18 & \\
\hline \multirow[t]{2}{*}{ HWE } & G. camprestris & * & * & * & * & * & * & NS & * & * & * & * & * & \\
\hline & G. bimaculatus-like & NS & * & * & NS & NS & NS & NS & * & * & * & NS & * & \\
\hline
\end{tabular}

Range G. camprestris 151-177 202-220 256-324 132-162 179-211 250-284 155-169 194-262 $185-195 \quad 201-215 \quad 289-323$

$(\mathrm{bp})^{1} \quad(167-197)(215-219)(262-317)(142-166)(180-192)(270-281)(163-178)(203-276)(187-197)(211-257)(303-320)$

G. bimaculatus-like $151-177 \quad 202-214 \quad 256-332 \quad 132-162 \quad 181-211 \quad 258-286 \quad 155-167 \quad 194-262 \quad 185-189 \quad 207-219 \quad 305-319$ $(150-220)(217-265)(246-387)(125-183)(180-241)(270-299)(157-181)(265-347)(206-240)(205-239)(310-437)$

${ }^{1}$ In parentheses: allele sizes observed for wild G. campestris and laboratory G. bimaculatus populations originating from Spain (Dawson et al., 2003; Bretman et al., 2008). Both populations were large and represented by 15-30 individuals. For locus Gbim58 that was excluded from statistical analysis, no differences in allele sizes ranges between these two cricket groups were observed in this study (G. campestris: 83-113 bp vs. G. bimaculatus-like: 89-111 bp), in contrary to the populations from Spain (G. campestris: 95-99 bp vs. G. bimaculatus: 114-149 bp).

statistically significant (Table 1). The allelic size ranges of single loci of the two crickets group were almost identical and in most cases in the range observed for G. campestris (Dawson et al., 2003; Bretman et al., 2008). In general, both groups exhibited very similar pattern across all calculated diversity indices. Estimated $F_{I S}$ values were positive and significant, indicating internal genetic structure (Wahlund effect).

Genetic polymorphism levels of the 13 cricket collections ( $n=262$ individuals), for which the sampled individuals number exceeded 15 , were generally higher than observed in the cricket populations in northern Germany (Witzenberger \& Hochkirch, 2008). The calculated values ranged for $N_{4}$ from 4.18 to 6.64 (in comparison to 3.75 5.75), for $N_{e}$ from 2.48 to 4.06 (compared to 2.07-4.22) and for $R$ from 4.08 to 5.77 (in comparison to 3.58-5.67). The observed differences in the genetic variability between the Polish and German populations are rather small, although the eastern populations seem to be more polymorphic, which is consistent with the abundances trends and population dynamic in both countries. The field cricket populations in north Germany have been greatly reduced in number and isolated, contrary to the situation in Poland, where populations remain stable and abundant (Bazyluk \& Liana, 1990, 2000; Hochkirch et al., 2007; Witzenberger \& Hochkirch, 2008). In our present study, the mean number of alleles in the 13 cricket populations sampled was, however, significantly lower compared to the G. campestris population from Asturias in Northern Spain $(\mathrm{n}=541)$ where $N_{A}$ was $\sim 11.4$ across 11 loci (Bretman et al., 2011). Interestingly, we obtained similar $N_{A}$ value when we pooled all of the studied crickets $(\mathrm{n}=414)$ sampled over sites occupying several dozens of square $\mathrm{km}$, while the population from North Spain inhabited only a $800 \mathrm{~m}^{2}$ meadow.

\section{Introgression assessment}

Admixture analyzes showed no clear subdivision of individuals possessing the two different haplotypes. Both $G$. campestris as well as G. bimaculatus-like individuals were recorded in the two clusters in STRUCTURE (Fig. 4); even so, the distribution of $G$. campestris and G. bimaculatuslike individuals in the two clusters was not random and the proportion of individuals assigned to cluster 1 represented $16 \%$ and $60 \%$ in case of G. bimaculatus-like and G. campestris individuals, respectively, the difference being highly significant (Chi-square test, $\chi_{1}^{2}=59.5, P<0.001$ ). Similarly, some genetic divergence was observed when pairwise genetic distances $\left(D_{A}\right)$ were calculated and expressed via PCoA (Fig. 5). Mean values of the scores of particular individuals along the first PCoA axis did not differ between the two species ( $t$-test, $t_{131}=0.48, P=0.631$ ). However, the two species were separable along the second PCoA axis:

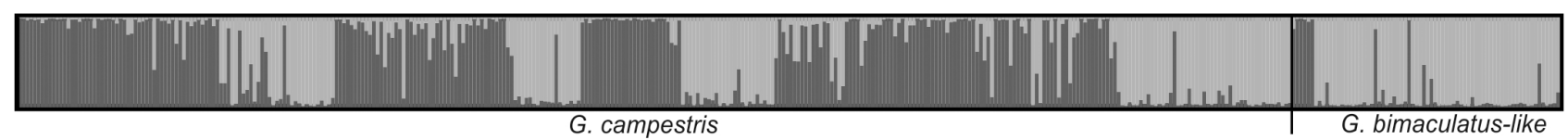

Fig. 4. Admixture analyses of 414 field crickets performed using STRUCTURE with $K=2$. Each individual is represented by a vertical bar. The vertical black line separates individuals belonging to the two distinct haplotype lineages. $K-$ number of clusters 


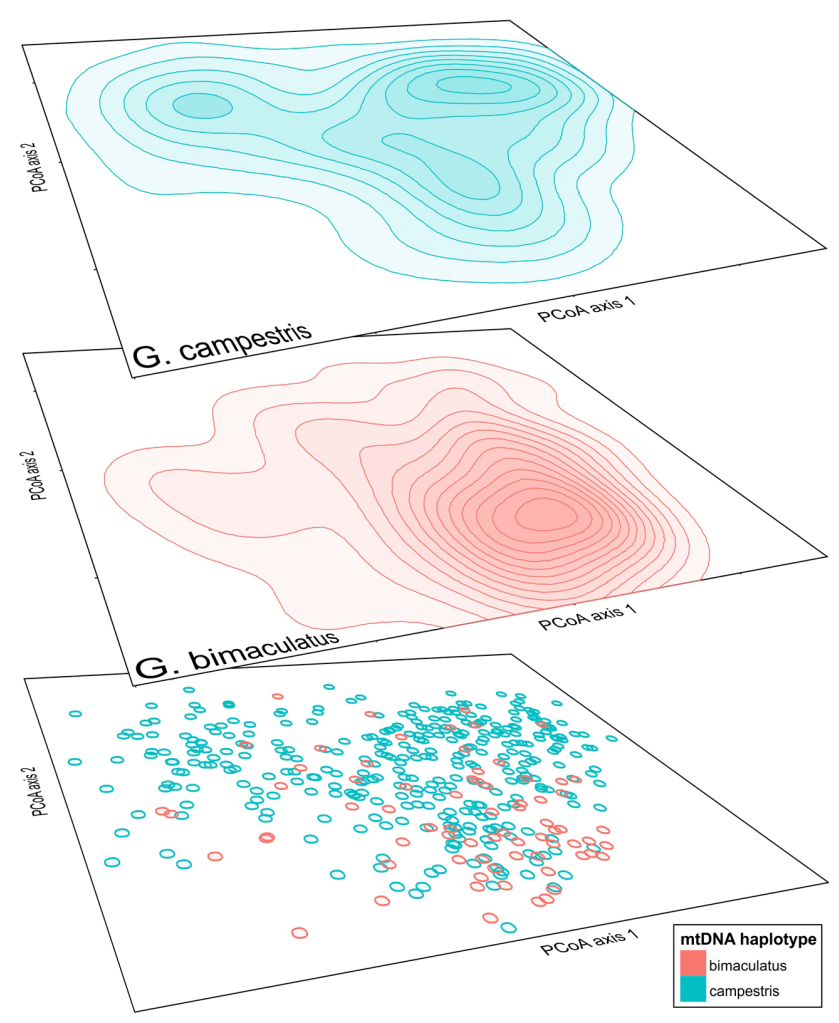

Fig. 5. Principal coordinate analyses (PCoA) of the 414 field crickets based on the matrix of $D_{A}$ distances of Nei et al. (1983). The two axes of PCoA explain $12 \%$ of the total variance of the distance. The bottom subplot presents the projections of particular individuals on the plot delimited by first and second axis of PCoA, middle and upper subplots present kernel density estimators for the $G$. campestris and G. bimaculatus-like specimens.

G. campestris individuals were associated with the higher score values compared to $G$. bimaculatus-like individuals (unequal variance $t$-test, $t_{100}=8.06, P<0.0001$ ). Among all the PCoA axes $(n=123)$, there were 12 cases showing significant differences $(P<0.05$, with no correction for the multiple comparisons) in the location between $G$. campestris and G. bimaculatus-like individuals. As here demonstrated, the G. campestris group is more genetically complex than hitherto assumed, forming at least three concentrations visible on the kernel density plot, whereas the G. bimaculatus-like individuals were contrastingly restricted to one.

Separate calculations of the two X-chromosome-linked loci, Gbim59 and Gbim71, showed a similar pattern as the autosomal loci, revealing an almost complete introgression of individuals carrying the G. bimaculatus-like haplotype into the $G$. campestris gene pool. Analyzes of males ( $\mathrm{n}=$ 261), for which these loci were treated as one unit in the haploid-STR way (males are X0), revealed the existence of seven different genotypes (Table 2). We followed the distributions of these genotypes across six populations representing "pure" G. campestris collections and compared them with 11 collections, where both mitochondrial haplotypes were recorded (separately for $G$. campestris and $G$. bimaculatus-like individuals). Most of the individuals bore the same genotype, regardless of the mitochondrial haplotype contribution. Differences in the proportions of genotypes in each group were statistically significant for two genotypes: thus genotype $134 / 88$ was recorded in $>12 \%$ males in the $G$. campestris group, regardless of whether the populations were pure or mixed, whereas in the G. bimaculatus-like individuals, this genotype was absent, as was genotype 136/82, which was more common in G. bimaculatus-like individuals compared to G. campestris (Table 2).

\section{DISCUSSION}

In this study, we investigated the variability of cytochrome $b$ gene fragment (mtDNA) and microsatellite loci (autosomal and sex-linked) of field crickets representing 24 different sub-populations from the Oder and Vistula River valleys of north-western Poland. The genetic diversity indices for the separate populations were rather low when compared to the old and large population of $G$. campestris in North Spain (Bretman et al., 2011), though higher than described for the depleted populations in northern Germany (Hochkirch et al., 2007). Mitochondrial sequence analyses revealed the occurrence of a $G$. bimaculatus-like haplotype, which was unexpected in this region. It seems that this introduced species has successfully introgressed into the gene-pool of local G. campestris populations, which is worrying, considering its endangered status in Western Europe.

TABLE 2. Genotype distribution of the two chromosome X-linked loci in males, belonging to the two field crickets groups of G. campestris and G. bimaculatuslike mitochondrial haplotypes. $n$ total $=230$ individuals without missing data consisting of 11 populations with mixed origin $(n=123 \mathrm{G}$. campestris and $n=$ 43 G. bimaculatus-like crickets) and 6 populations of pure G. campestris individuals $(n=64)$. Results of the Chi-square test (test statistics and $P$ values) comparing genotype frequencies between groups of crickets are given in the last two columns: "y" denotes Yates continuity correction applied, significant differences are bolded.

\begin{tabular}{|c|c|c|c|c|c|c|c|}
\hline & \multicolumn{2}{|c|}{ Genotype } & \multicolumn{2}{|c|}{ Eleven "mixed" populations (\%) } & \multirow{2}{*}{$\begin{array}{c}\text { Six "pure" populations (\%) } \\
{[\mathrm{C}]} \\
\text { G. campestris }\end{array}$} & \multicolumn{2}{|c|}{ Differences - chi-square test } \\
\hline & Gbim711 & Gbim59 & $\begin{array}{c}{[\mathrm{A}]} \\
\text { G. campestris }\end{array}$ & $\begin{array}{c}{[\mathrm{B}]} \\
\text { G. bimaculatus-like }\end{array}$ & & {$[A]$ vs. $[B]$} & {$[\mathrm{A}]$ vs. $[\mathrm{C}]$} \\
\hline 1 & 134 & 82 & 81.30 & 76.74 & 79.69 & $X^{2}=0.41 ; P=0.519$ & $X^{2}=0.07 ; P=0.790$ \\
\hline 2 & 134 & 88 & 12.20 & 0.00 & 12.50 & $\mathrm{X}^{2}=4.38 ; P=0.036 \mathrm{y}$ & $X^{2}=0.01 ; P=0.952$ \\
\hline 3 & 140 & 82 & 0.81 & 0.00 & 3.13 & $\mathrm{X}^{2}=0.30 ; P=0.581 \mathrm{y}$ & $X^{2}=0.34 ; P=0.561 y$ \\
\hline 4 & 140 & 88 & 0.00 & 0.00 & 3.13 & - & $X^{2}=1.49 ; P=0.222 y$ \\
\hline 5 & 136 & 88 & 0.00 & 4.65 & 1.56 & $x^{2}=2.54 ; P=0.111 y$ & $\mathrm{X}^{2}=0.11 ; P=0.734 \mathrm{y}$ \\
\hline 6 & 132 & 82 & 3.25 & 6.98 & 0.00 & $\mathrm{X}^{2}=0.34 ; P=0.545 \mathrm{y}$ & $\mathrm{X}^{2}=0.86 ; P=0.354 \mathrm{y}$ \\
\hline 7 & 136 & 82 & 2.44 & 11.63 & 0.00 & $\mathrm{X}^{2}=4.03 ; P=0.045 \mathrm{y}$ & $X^{2}=0.42 ; P=0.518 y$ \\
\hline
\end{tabular}

${ }^{1}$ Allele sizes for both loci observed in wild G. campestris and laboratory G. bimaculatus populations (Dawson et al., 2003; Bretman et al., 2008) were similar for both species and in this study. 
Gryllus bimaculatus occurs in Europe predominantly in the Mediterranean areas, in Africa and Asia in tropical and subtropical regions (Heller et al., 1998; Bazyluk \& Liana, 2000; Ferreira \& Ferguson, 2010). Ferreira \& Ferguson (2010) suggest that the distribution and abundance of this species in Europe is shaped by migration from warmer regions (mainly from Africa) and limited toward the north by the winter temperature isotherm around $16^{\circ} \mathrm{C}$. Until now, no natural $G$. bimaculatus population has been described in Poland or other regions of northern Europe. The occurrence of G. bimaculatus-like crickets in north-western Poland (lower Odra River valley), whilst unexpected, must nevertheless be viewed in the light of the fact that $G$. bimaculatus was recently recorded in a few localities both in Germany and France (following the Fauna Europea website: http://www.faunaeur.org/distribution_table.php). It is thus possible that the presence of xerothermic vegetation in the region facilitates the persistence of this thermophilous cricket's population, but habitat preferences of the two species needs further investigations. Moreover, winter temperatures are much lower than the G. bimaculatus survival threshold proposed by Ferreira \& Ferguson (2010).

Several scenarios may explain the presence of the $G$. bimaculatus-like haplotype in $G$. campestris populations. It could result from incomplete lineage sorting during speciation or the maintenance of an ancestral polymorphism. However, in such a scenario, one might expect random distribution of the mtDNA haplotypes in the studied populations. The observed eastern-western gradient of $G$. bimaculatus-like haplotype frequencies, given the limited mobility of crickets, is therefore consistent with the scenario of a recent introduction of a small number of individuals into the natural population. It is possible that an artificiallyintroduced species has successfully introgressed into the natural $G$. campestris populations. This explains the high frequency of the mtDNA haplotype $G$. bimaculatus which, due to climatic conditions, could not survive winters in that part of Europe. In light of this, it seems reasonable to conclude that a group of $G$. bimaculatus-like individuals was accidentally or intentionally (probably the former) introduced into the wild and thanks to propitious circumstances intermixed into local populations of $G$. campestris. This view is supported by the fact that $G$. bimaculatus is commonly kept as food for spiders and reptiles, as well as being a model species used in scientific research (Simmons, 1986, 1991).

The analysis of microsatellite loci provides additional support for these assumptions, as individuals carrying the G. bimaculatus-like haplotype did not form a separate cluster, but were grouped together with $G$. campestris crickets. This means that the observed intermixing likely lasted for at least several generations. The genetic diversity indices and heterozygosity levels between the two cricket groups differed only slightly. In the study of Bretman et al. (2008), where individuals representing pure G. bimaculatus or $G$. campestris species were analysed, allelic ranges of several microsatellite loci (i.e. Gbim49, 58, 72, 33 and 66) were different and only partially overlapping for the two species.
In contrast, in our study, the observed allelic size ranges were almost identical, indicating advanced intermixing. However, the spatial genetic structure seems to be still affected by the observed introgression, as the probability of successful prediction of a randomly chosen individual carrying $G$. bimaculatus-like haplotype to the second cluster (at the uppermost level of substructure) was high.

It was proven that gene-flow between the two cricketspecies is asymmetrical with $G$. campestris females almost never hybridizing. In contrast, $G$. bimaculatus females hybridize more readily, which is explained by their higher degree of polyandry (Veen et al., 2011, 2013; Tyler et al., 2013). Hybrid females prefer mating with $G$. bimaculatus males, while hybrid males have reduced attractiveness in general, as fact that should restrict further gene flow (Veen et al., 2013). These particular studies showed that in the case of hybridization, the interspecific introgression should occur mainly through $G$. bimaculatus females and G. campestris males (Veen et al., 2011, 2013). In such circumstances, the mtDNA haplotype of a relatively small group of introduced individuals could spread within the natural population due to genetic drift. Similar conclusions were made by Hochkirch \& Lemke (2011) for asymmetrical hybridization in the case of other Orthoptera species, i.e. Chorthippus montanus and C. parallelus (Orthoptera: Acrididae).

Knowing that introgression under laboratory conditions is asymmetric and that hybrid males are much less attractive to females than paternal species (Veen et al., 2011, 2013), means that different genotypes distributions on the X-chromosome-linked markers between individuals carrying the two mitochondrial haplotypes are expected. We wished to test if any signs of reduced gene flow could be observed, especially as it is generally accepted the $\mathrm{X}$ chromosome is very important in the evolution of reproductive isolation (reviewed by Turelli \& Moyle, 2007; Bolnick et al., 2008; Good et al., 2008). We compared these patterns between males, as they possess only one $\mathrm{X}$ chromosome, inherited from their mothers. Indeed, the G. campestris males had one genotype present both in pure and intermixed populations with similar, quite high frequency $(>10 \%)$, that was absent in the G. bimaculatus-like individuals. The observed differences of the X-chromosome-linked loci between the G. campestris and G. bimaculatus-like groups were, however, weak (although significant) and masked probably by several generations of interbreeding. Such differences may though serve as a signal that the observed intermixing is an ongoing process in the studied populations of field cricket.

When able to choose, both species (and both sexes) strongly prefer conspecifics as mates (Tyler et al., 2013; Veen et al., 2013). Interbreeding avoidance could be even stronger in natural contact zones, where both species have co-existed in sympatry for a long time and the species may develop additional behavioral mechanisms which reinforce ecological-evolutionary divergence. For example, G. fultoni (Alexander) differs in calling songs depending on if it occurs in sympatry or allopatry with $G$. vernalis Blatchley (Jang \& Gerhard, 2006). However, in artificially created 
contact zones, the native species usually outnumbers the introduced one, facilitating hybridization. As shown in this study, costs of hybridization are sufficiently low to enable gene-flow at least through the females of G. bimaculatus. Backcrossing with parental G. campestris must be unavoidable and seems not to affect the $\mathrm{F}_{2}$ fitness in preventing further reproduction. This will probably result in persistence of the hetero-specific mitochondrial haplotype in the next generations. The important issue that needs to be quantified is the autosomal contribution of $G$. bimaculatus to the $G$. campestris gene pool, as it have been shown that this may be negligible even when introgression of mitochondrial DNA has occurred due to sex-biased asymmetries or/and adaptive forces causing mito-nuclear discordances (Toews \& Brelsford, 2012; Good et al., 2015).

The phenomenon observed in this study emphasizes the threat for both the numerous and stable populations of field crickets in Poland, as well as the declining and endangered populations in Germany, essentially because of the continuing diffusion of $G$. bimaculatus genes, which is highly probable. The genetic integrity of $G$. campestris as a species has been locally disrupted and the surrounding, currently unaffected populations are at the same risk. What is more, occasional reports of $G$. bimaculatus in Germany and France indicate that the problem of non-intentional introductions of $G$. bimaculatus and the species' introgression into native populations of G. campestris may also have taken place in other regions of western Europe. In theory, $G$. campestris and $G$. bimaculatus can be easily distinguished morphologically (i.e. width of the head in relation to body, length of wings, size of yellow patches and general body size; Bazyluk, 1956). There are also some phenological differences in the activity of the two species during the year $-G$. campestris is in general active earlier than G. bimaculatus (Bellman, 2006). Having said that, three randomly selected individuals from the groups of crickets carrying $G$. bimaculatus-like haplotypes were morphologically determined by an Orthoptera expert to be G. campestris (A. Liana, pers. comm.), showing that genetic evaluation is necessary in this case.

The introgression of genes from non-native species is a well-known phenomenon (cf. Crispo et al., 2011 and references therein), yet the effects are poorly understood. It can decrease adaptation and affect fitness of the local populations. The impact of the observed introgression of G. bimaculatus-like individuals into natural populations of G. campestris, should therefore be characterized, especially by quantifying the extent of nuclear gene flow between these species through collection of genome-wide data. Our findings seem to be especially important in the light of restitution efforts, including translocations of $G$. campestris (Witzenberger \& Hochkirch, 2008), more especially as introductions of $G$. bimaculatus individuals may negatively influence the restitution process. Populations scheduled for use as a source for reintroductions should be first genetically characterized to exclude possible introgression. Populations proven to be already introgressed with $G$. $b i$ maculatus-like haplotypes should not be used for translo- cations even if their genetic parameters like heterozygosity or effective population sizes are high.

ACKNOWLEDGEMENTS. We are grateful to the anonymous reviewers for their valuable comments. This study was supported by National Science Center grant no. NN303322234 directed by M. Żmihorski and the project financing agreements POIG.02.02.0014-024/08-00. We are grateful to K. Barańska and M. Molak for field work assistance. English proofreading of the manuscript was kindly performed by B. Przybylska and A. Baca, whilst H.D. Loxdale also made valuable editorial suggestions for the improvement of the manuscript.

\section{REFERENCES}

Andrés J.A., Larson E.L., Bogdanowicz S.M. \& Harrison R.G. 2013: Patterns of transcriptome divergence in the male accessory gland of two closely related species of field crickets. Genetics 193: 501-513.

BAZYLuK W. 1956: Klucze do oznaczania owadów Polski, XI Prostoskrzydte - Orthoptera (Saltatoria). [A Key for the Identification of Insects of Poland XI, Orthoptera (Saltatoria).] PWN, Warsaw, 166 pp.

Bazyluk W. \& Liana A. 1990: Orthoptera (Saltatoria) Prostoskrzydłe. In Razowski J. (eds): Wykaz zwierzat Polski, Tom I. [List of Animals of Poland, Vol. 1.] Wydawnictwo Polskiej Akademii Nauk, Ossolineum, Wrocław, Warszawa, Kraków, 4751 pp.

Bazyluk W. \& Liana A. 2000: Katalog Fauny Polski, Prostoskrzydte - Orthoptera, XVII, 2. Muzeum i Instytut Zoologii PAN, Warsaw, $156 \mathrm{pp}$.

Bellman H. 2006: Der Kosmos Heuschreckenführer. Die Arten Mitteleuropas sicher bestimmen. Franckh-Kosmos, Stuttgart, $355 \mathrm{pp}$.

Bolnick D.I., Turelli M., López-Fernández H., Wainwright P.C. \& NeAR T.J. 2008: Accelerated mitochondrial evolution and "Darwin's corollary": asymmetric viability of reciprocal F1 hybrids in Centrarchid fishes. - Genetics 178: 1037-1048.

Bretman A., Dawson D.A., Horsburgh G.J. \& Tregenza T. 2008: New microsatellite loci isolated from the field cricket Gryllus bimaculatus characterized in two cricket species, Gryllus bimaculatus and Gryllus campestris. - Mol. Ecol. Resour. 8: 1015-1019.

Bretman A., Rodríguez-Muñoz R., Walling C., Slate J. \& TreGENZA T. 2011: Fine-scale population structure, inbreeding risk and avoidance in a wild insect population. - Mol. Ecol. 20: 3045-3055.

Crispo E., Moore J.S., Lee Yaw J.A., Gray S.M. \& Haller B.C. 2011: Broken barriers: Human-induced changes to gene flow and introgression in animals. - BioEssays 33: 508-518.

Dawson D.A., Bretman A.J. \& Tregenza T. 2003: Microsatellite loci for the field cricket, Gryllus bimaculatus and their crossutility in other species of Orthoptera. - Mol. Ecol. Notes 3: 191-195.

Dieringer D. \& Schlötterer C. 2003: Microsatellite analyser (MSA): a platform independent analysis tool for large microsatellite data sets. - Mol. Ecol. Notes 3: 167-169.

EARL D.A. \& von Holdt B.M. 2012: Structure Harvester: a website and program for visualizing STRUCTURE output and implementing the Evanno method. - Conserv. Genet. Resour. 4: $359-361$.

Evanno G., Regnaut S. \& Goudet J. 2005: Detecting the number of clusters of individuals using the software STRUCTURE: a simulation study. - Mol. Ecol. 14: 2611-2620. 
EXCOFFIER L. \& Lischer H.E.L. 2010: Arlequin suite ver 3.5: A new series of programs to perform population genetics analyses under Linux and Windows. - Mol. Ecol. Resour. 10: 564-567.

Ferreira M. \& Ferguason J.W.H. 2010: Do Mediterranean crickets Gryllus bimaculatus De Geer (Orthoptera: Gryllidae) come from the Mediterranean? Largescale phylogeography and regional gene flow. - Bull. Entomol. Res. 100: 49-58.

Good J.M., Dean M.D. \& Nachman M.W. 2008: A complex genetic basis to X-linked hybrid male sterility between two species of house mice. - Genetics 179: 2213-2228.

Good J.M., Vanderpool D. \& Keeble S. \& Bi K. 2015: Negligible nuclear introgression despite complete mitochondrial capture between two species of chipmunks. - Evolution 69: 1961-7192.

Goudet J. 2002: Fstat, Ver. 2.9.3.2. Institute of Ecology, Lausanne. http://www2.unil.ch/izea/softwares/fstat.html

Grein G. 2000: Zur Verbreitung der Heuschrecken (Saltatoria) in Niedersachsen und Bremen. - Informationsdienst Natursch. Niedersachs. 20: 74-112.

GREIN G. 2005: Rote Liste der in Niedersachsen und Bremen gefahrdeten Heuschrecken mit Gesamtartenverzeichnis 3. Fassung. - Informationsdienst Natursch. Niedersachs. 25: 1-20.

HaLl T.A. 1999: BioEdit: a user-friendly biological sequence alignment editor and analysis program for Windows 95/98/NT. — Nucl. Acids Symp. Ser. 41: 95-98.

HARRISON R.G. 1983: Barriers to gene exchange between closely related cricket species. I. Laboratory hybridization studies. Evolution 37: 245-251.

HARRISON R.G. 1986: Pattern and process in a narrow hybrid zone. - Heredity 56: 337-349.

Harrison R.G., Rand D.M. \& WheEler W.C. 1987: Mitochondrial DNA variation in field crickets across a narrow hybrid zone. - Mol. Biol. Evol. 4: 144-158.

Heller K.G., Korsunovskaya O., Ragge D.R., Vedenina V., WilLEMSE F., Zhantiev R.D. \& Frantsevich L. 1998: Check-list of European Orthoptera. - Articulata 7: 1-61.

HochKirch A. \& Lemke I. 2011: Asymmetric mate choice, hybridization, and hybrid fitness in two sympatric grasshopper species. - Behav. Ecol. Sociobiol. 65: 1637-1645.

Hochkirch A., Witzenberger K.A., Teerling A. \& Niemeyer F. 2007: Translocation of an endangered insect species, the field cricket (Gryllus campestris Linnaeus, 1758) in northern Germany. - Biodivers. Conserv. 16: 3597-3607.

Holusa J. 2012: Grasshoppers and bushcrickets regionally extinct in the Czech Republic: consequence of the disappearance of habitats scattered on the edge of their ranges. - J. Insect Conserv. 16: 949-960.

Holusa J., Kocarek P., Marhoul P. \& Skokanova H. 2012: Platycleis vittata (Orthoptera: Tettigoniidae) in the northwestern part of its range is close to extinction: is this the result of landscape changes? - J. Insect Conserv. 16: 295-303.

Huang Y., Ortí G., Sutherlin M., Duhachek A. \& Zera A. 2000: Phylogenetic relationships of north American field crickets inferred from mitochondrial DNA data. - Mol. Phylogenet. Evol. 17: 48-57.

JAKOBSSON M. \& RoSENBERG N.A. 2007: CLUMPP: a cluster matching and permutation program for dealing with label switching and multimodality in analysis of population structure. - Bioinformatics 23: 1801-1806.

JANG Y. \& GeRhaRDT H.C. 2006: Divergence in the calling songs between sympatric and allopatric populations of the southern wood cricket Gryllus fultoni (Orthoptera: Gryllidae). - J. Evol. Biol. 19: 459-472.
Larson E.L., Hume G.L., Andrés J.A. \& Harrison R.G. 2012: Post-mating prezygotic barriers to gene exchange between hybridizing field crickets. - J. Evol. Biol. 25: 174-186.

Maroja L.S., Andrés J.A., Walters J.R. \& Harrison R.G. 2009: Multiple barriers to gene exchange in a field cricket hybrid zone. - Biol. J. Linn. Soc. 97: 390-402.

Maroja L., McKenzie Z.M., Hart E., Jing J., Larson E.L. \& RICHARDSON D.P. 2014: Barriers to gene exchange in hybridizing field crickets: the role of male courtship effort and cuticular hydrocarbons. - BMC Evol. Biol. 14: 65.

Nei M., Tajima F. \& Tateno Y. 1983: Accuracy of estimated phylogenetic trees from molecular data. - J. Mol. Evol. 19: 153-170.

Paekal R. \& Smouse P.E. 2006: GENALEX 6: genetic analysis in Excel. Population genetic software for teaching and research. - Mol. Ecol. Notes 6: 288-295.

Panagiotopoulou H., Baca M., Baca K., Stanoković A. \& ŻMIHORSKI M. 2015: Optimization and validation of a multiplex assay for microsatellite loci analysis in the field cricket, Gryllus campestris (Orthoptera: Gryllidae). - J. Asia Pac. Entomol. 18: 421-424.

Pritchard J.K., Stephens M. \& Donnelly P. 2000: Inference of population structure using multilocus genotype data. - Genetics 155: 945-959.

R CoRe TeAm 2015: R: A Language and Environment for Statistical Computing. R Foundation for Statistical Computing, Vienna, http://www.R-project.org/

RaYMOND M. \& Rousset F. 1995: Genepop (version 1.2): population genetics software for exact tests and ecumenicism. $-J$. Hered. 86: 248-249.

Ritz M.S. \& KöHLER G. 2007: Male behaviour over the season in a wild population of the field cricket Gryllus campestris L. Ecol. Entomol. 32: 384-392.

Rodríguez-Muñoz R., Bretman A., Slate J., Walling C.A. \& TregenzA T. 2010: Natural and sexual selection in a wild insect population. - Science 328: 1269-1272.

RONQUIST F. \& HUELSENBECK J.P. 2003: MRBAYES 3: Bayesian phylogenetic inference under mixed models. - Bioinformatics 19: $1572-1574$.

RosENBERG N.A. 2004: DISTRUCT: a program for the graphical display of population structure. - Mol. Ecol. Notes 4: 137138.

Simmons L.W. 1986: Female choice in the field cricket, Gryllus bimaculatus (de Geer). — Anim. Behav. 34: 1463-1470.

Simmons L.W. 1991: Female choice and the relatedness of mates in the field cricket, Gryllus bimaculatus. - Anim. Behav. 41: 493-501.

Tamura K., Peterson D., Peterson N., Stecher G., Nei M. \& KumAR S. 2011: MEGA5: Molecular evolutionary genetics analysis using maximum likelihood, evolutionary distance, and maximum parsimony methods. - Mol. Biol. Evol. 28: 2731-2739.

Toews D.P. \& Brelsford A. 2012: The biogeography of mitochondrial and nuclear discordance in animals. - Mol Ecol. 21: 3907-3930.

Tryjanowski P., Hartel T., Baldi A., Szymański P., TobóŁKa M., Herzon I., Golawski A., Konvicka M., Hromada M., Jerzak L., Kujawa K., Lenda M., OrŁowski G., Panek M., Skórka P., Sparks T.H., Tworek S., WucZyŃSKi A. \& ŻMIHORSKi M. 2011: Conservation of farmland birds faces different challenges in Western and Central-Eastern Europe. - Acta Ornithol. 46: $1-12$.

Turelli M. \& Moyle L.C. 2007: Asymmetric postmating isolation: Darwin's corollary to Haldane's rule. - Genetics 176: 1059-1088. 
Tyler F., Harrison X.A., Bretman A., Veen T., Rodríguez-MuÑOZ R. \& TregenZA T. 2013: Multiple post-mating barriers to hybridization in field crickets. — Mol. Ecol. 22: 1640-1649.

Untergasser A., Cutcutache I., Koressaar T., Ye J., Faircloth B.C., Remm M. \& Rozen S.G. 2012: Primer3 - New capabilities and interfaces. - Nucl. Acids Res. 40(15): e115, 12 pp.

Van Oosterhout C., William F., Hutchinson D.P., Wills M. \& SHIPLEY P. 2004: Micro-Checker: software for identifying and correcting genotyping errors in microsatellite data. - Mol. Ecol. Notes 4: 535-538.

Veen T., Faulks J., Tyler F., Lloyd J. \& Tregenza T. 2011: Premating reproductive barriers between hybridising cricket spe- cies differing in their degree of polyandry. - PLOS ONE 6(5): e19531, 7 pp.

Veen T., Faulks J., Tyler F., Lloyd J. \& Tregenza T. 2013: Diverse reproductive barriers in hybridising crickets suggests extensive variation in the evolution and maintenance of isolation. - Evol. Ecol. 27: 993-1015.

WitZENBERger K.A. \& HochKIRCH A. 2008: Genetic consequences of animal translocations: A case study using the field cricket, Gryllus campestris L. — Biol. Conserv. 141: 3059-3068.

Received November 9, 2015; revised and accepted May 25, 2016 Published online August 29, 2016 